\title{
EFEITOS MULTIPLICADORES DA ECONOMIA FLORESTAL BRASILEIRA ${ }^{1}$
}

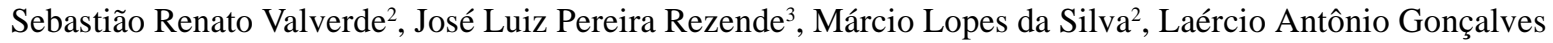 \\ Jacovine $^{2}$ e Rosa Maria Miranda Armond Carvalho ${ }^{4}$
}

\begin{abstract}
RESUMO - Este trabalho trata da importância do setor florestal para o desenvolvimento socioeconômico brasileiro por meio dos modelos econômicos de equilíbrio geral multissetoriais, através das análises das matrizes de insumoproduto (MIP) e de contabilidade social (MCS). As principais fontes dos dados foram as Tabelas de InsumoProduto (TIP) do Instituto Brasileiro de Geografia e Estatística (IBGE), além de informações obtidas no Banco Nacional de Desenvolvimento Econômico e Social (BNDES), no Banco Central (BACEN) e na Secretaria da Receita Federal (SRF), referentes ao ano de 1995. Observou-se que tão importante quanto contribuir para a geração e formação do PIB, de emprego, impostos, salários e balança comercial é o poder que um setor produtivo tem a mais que o outro de multiplicar estes indicadores quando uma unidade monetária é gasta a mais pelos consumidores finais na demanda por produtos deste determinado setor. Os resultados indicaram que o setor florestal é um dos setores da economia brasileira que apresentou os maiores efeitos multiplicadores para os indicadores socioeconômicos, sendo superiores aos de outros como o da indústria automobilística, de equipamentos elétricos e eletrônicos, de máquinas e equipamentos e de produtos químicos e petróleo.
\end{abstract}

Palavras-chave: Economia florestal, equilíbrio econômico e planejamento florestal.

\section{MULTIPLIER EFFECTS OF THE BRAZILIAN FORESTRY ECONOMY}

\begin{abstract}
This study evaluated the important role the forestry sector plays in the Brazilian economic development by means of the multisector general economic equilibrium models, through the analyses of the Input-Output Matrix (IOM) and the Social Accounting Matrix (SAM). The main sources were the Input-Output Tables (IOT) from the Brazilian Institute of Geography and Statistics (IBGE), the National Bank of Economic and Social Development (BNDES), from the Brazilian Central Bank (BACEN) and the Brazilian Federal Revenue Office $(S R F)$, for 1995. It was observed that the extra power a productive sector has over another to multiply GDP, jobs, taxes, salaries and trade balance, is as important as contributing for the generation of these indicators, when one extra monetary unit is spent by the final consumers demanding for products of this particular sector. The results showed that forestry is one of the Brazilian economic sectors presenting the greatest multiplying effects for the social-economic indicators. It surpassed other sectors such as the automobilistic electric and electronic, machinery and equipment and chemicals and petroleum industries.
\end{abstract}

Key words: Forestry economy, economic equilibrium and forestry planning.

\section{INTRODUÇÃO}

A economia florestal brasileira tem uma participação significativa nos indicadores socioeconômicos do País, como o Produto Interno Bruto (PIB), empregos, salários, impostos e balança comercial. No mercado internacional de produtos florestais como a celulose, madeira, móveis, laminados etc., o Brasil vem conquistando espaço em razão das vantagens competitivas que possui.

1 Recebido para publicação em 29.1.2002.

Aceito para publicação em 12.5.2003.

2 Professor do Dep. de Engenharia Florestal da Universidade Federal de Viçosa - UFV, 36571-000 Viçosa-MG. ${ }^{3}$ Professor do Dep. de Ciências Florestais da Universidade Federal de Lavras - UFLA, 37200-000 Lavras-MG. ${ }^{4}$ Doutoranda em Ciência Florestal, Dep. de Engenharia Florestal da UFV. 
Em termos sociais, o setor florestal vem se destacando por absorver grande parte dos trabalhadores dispensados por outras atividades econômicas, principalmente da agricultura e de manufaturados. Nas regiões montanhosas onde a agricultura está sendo desestimulada, o setor florestal se apresenta como alternativa para os produtores e trabalhadores rurais, visto que estas regiões ainda conservam toda uma aptidão florestal.

Diante do papel social do setor florestal brasileiro, de sua vantagem competitiva e de sua capacidade de alavancar o crescimento socioeconômico, é importante desenvolver um estudo que mostre estas peculiaridades e que possa ser utilizado como referência na elaboração de políticas que visem favorecer o desenvolvimento regional.

Os modelos econômicos ideais para auxiliar neste estudo são os de equilíbrio econômico geral, como a matriz de insumo-produto (MIP), a matriz de contabilidade social (MCS) e os modelos computáveis de equilíbrio geral (CEG).

O uso desses modelos depende de um sistema de contas integradas que descreva todos os processos de produção, circulação, consumo e acumulação na economia, denominado Sistemas de Contas Nacionais (SCN). Tais modelos servem para quantificar os choques exógenos no sistema econômico de forma mais completa e realista que os modelos abstratos (Murray, 1995; Weintraub \& Cholaky, 1991).

A MIP representa o lado produtivo da economia com suas relações intersetoriais. É uma adaptação simplificada do modelo neoclássico de equilíbrio geral, especialmente do modelo walrasiano. Nas suas colunas são lidas as despesas e nas linhas, as receitas.

A MCS descreve o fluxo circular completo de uma economia. Ela fornece um conjunto completo e consistente de informações sobre todas as transações entre os setores e agentes em uma economia. A MCS é representada por uma matriz quadrada. As transferências de renda de um agente (coluna) para outro (linha), na MCS, formam o fluxo circular da economia que está sendo representada (Vieira, 1998). Suas origens estão na MIP, da qual constituem uma versão ampliada, ao agregar aos coeficientes técnicos de produção outras informações acerca da produção, da remuneração dos fatores e de estrutura da demanda (intermediária e final), desagregando-as por setores e grupos socioeconômicos (Castilho, 1994).

R. Árvore, Viçosa-MG, v.27, n.3, p.285-293, 2003
Os CEGs são modelos dinâmicos, capazes de simular o funcionamento de uma economia de mercado. Eles são sucessores das MIPs e MCSs, caracterizandose por permitir a substituição entre fatores e produtos na oferta e na demanda, além de introduzir um sistema de preços de mercado e uma completa especificação dos fluxos de renda e produto (Najberg et al., 1995).

Dos modelos econômicos de equilíbrio geral é possível obter os multiplicadores de impacto para saber o quanto um setor influencia o restante da economia quando sofre aumentos na demanda de seus produtos. Estes impactos vão refletir em variações na renda, no PIB, nos empregos gerados, nos impostos arrecadados e na balança comercial.

$\mathrm{O}$ efeito que o aumento da produção de um setor causa a outros setores, devido ao crescimento na demanda de seus produtos por parte do governo e dos consumidores finais, e que resulta em variações na renda, no emprego, na produção, nos impostos e no saldo da balança comercial é chamado de multiplicadores de impactos econômicos.

Se a demanda por madeira cresce em um dólar, a produção da economia crescerá em mais de um dólar. Porque para atender à demanda de seus produtos a indústria madeireira cria demanda por produtos de seus fornecedores, aumentando também a sua produção, e assim por diante. Cada ligação nessa cadeia produtiva adiciona um pouco mais para a produção da economia e, para produzir mais, pessoas trabalham mais, alugam mais espaços e emprestam mais capital para as indústrias, efeitos estes que podem ser calculados pelos multiplicadores da renda e do emprego.

Os multiplicadores de emprego para a indústria de produtos madeireiros consistem na mudança no emprego total da economia, causada por uma mudança unitária na demanda final por produtos madeireiros, dividido pelo emprego direto por unidade de produto na indústria de produtos madeireiros.

Muitos estudos nos quais são aplicados modelos econômicos de equilíbrio geral na economia florestal são realizados no mundo, exceto no Brasil, onde poucos são encontrados. Flick \& Teeter (1988) analisaram os multiplicadores econômicos para as indústrias de base florestal para oito estados da região sul dos Estados Unidos, derivados das MIPs, e observaram que os multiplicadores da produção, da renda e do emprego para a indústria florestal são maiores que a média dos demais setores dessa região. 
Valverde et al. (1997), trabalhando com modelo CEG, observaram, por meio de simulações em cenários de abertura comercial do Mercosul, da Rodada Uruguai e da Alca, que o Brasil obteria ganhos no bem-estar social, na produção e nas exportações de produtos florestais com a globalização dos mercados.

Nesse contexto, os objetivos deste trabalho foram determinar os impactos na produção, nos impostos, no emprego, nos salários e na balança comercial, por meio de choques nas demandas dos consumidores finais de cada setor analisado, e avaliar suas relações com seus fornecedores e consumidores intermediários, empregando os modelos econômicos multissetoriais MIP e MCS.

\section{MATERIAL E MÉTODOS}

Para execução dos objetivos deste trabalho, foram empregados os modelos econômicos de equilíbrio geral multissetoriais através das análises dos multiplicadores de impacto das MIP e MCS.

\subsection{Fonte dos Dados}

Os dados para este estudo referem-se ao ano de 1995 e são provenientes das tabelas de insumo-produto (TIP) fornecidas pelo Instituto Brasileiro de Geografia e Estatística (IBGE), do Banco Central (BACEN), do Banco Nacional de Desenvolvimento Econômico e Social (BNDES) e da Secretaria da Receita Federal (SRF).

As TIPs de um Sistema de Contas Nacionais (SCN) são formadas pelas tabelas de oferta de bens e serviços, de consumo intermediário, de demanda final, da conta de produção e distribuição operacional da renda e da produção.

Antecipando as discussões dos resultados, cabe descrever dois pontos importantes do trabalho, que são o cenário macroeconômico de 1995 e a forma como o IBGE agrega nas TIPs os produtos e as atividades florestais.

A conjuntura econômica em 1995 não era tão favorável ao setor florestal como a atual. Naquela época, o câmbio era prejudicial, pois durante muito tempo o real permaneceu igual e maior que o dólar e o processo de abertura comercial estava começando, o que afetava significativamente o volume das exportações brasileiras de produtos florestais.

Vale ressaltar que a utilização dos dados de 1995 deve-se ao fato de este ter sido o último ano em que as
TIPs do IBGE foram constituídas de informações observadas. A partir daí, o que se tem são projeções feitas principalmente em cima de variações no PIB. Sendo assim, levando-se em conta que as mudanças significativas que ocorreram no câmbio e na abertura comercial desde então não foram consideradas nessas projeções, concluise não ser problemática a utilização desta referência.

Quanto à forma de agregação das TIPs pelo IBGE, observa-se que muitos produtos florestais, como o carvão vegetal, a madeira em tora etc., são agregados como Outros Produtos Agropecuários, provocando a superestimação da atividade Agropecuária em detrimento da atividade Florestal. Outro ponto diz respeito à forma como IBGE agrega a atividade de siderurgia, pois esta, podendo usar carvão vegetal ou mineral, é agregada indistintamente em Siderurgia, o que para as entidades da classe produtiva florestal é incorreto, dado à participação, tanto em relação à quantidade quanto ao custo, do carvão vegetal na produção do ferro-gusa.

\subsection{Agregação das Atividades em Setores}

Neste trabalho, as 42 atividades e os 80 produtos contidos nas TIPs foram agregados em 13 setores (Quadro 1). Como o principal setor a ser analisado é o Florestal, procurou-se agregar as atividades em função da inter-relação (demanda e oferta) do setor Florestal com os demais setores consumidores e fornecedores de seus produtos.

\subsection{Modelo Econômico}

Utilizou-se a MIP para determinar os multiplicadores de impacto sobre a produção, o emprego, os salários, os impostos e a balança comercial, assim como a MCS para determinar os multiplicadores de efeitos global, transferência, cruzado, circular e totais (produção, valor adicionado e renda das instituições).

\subsection{Modelos Analíticos para Cálculo dos Multiplicadores}

Os fluxos intersetoriais de bens e serviços de uma economia, determinados por fatores tecnológicos e econômicos, são descritos por um sistema de equações simultâneas da seguinte forma:

$$
\mathrm{x}=\mathrm{Ax}+\mathrm{y}
$$


em que $\mathrm{x}=$ vetor $(\mathrm{nx} 1)$ com o produto total de cada setor; $\mathrm{y}=$ vetor ( $\mathrm{nx} 1)$ com a demanda final setorial; e A = matriz ( $\mathrm{n} \times \mathrm{n}$ ) com os coeficientes técnicos de produção.

Nesse modelo, com o vetor de demanda final setorial conhecido e fixo, pode-se determinar o vetor de produto total setorial através da seguinte equação matricial:

$$
\mathrm{x}=(1-\mathrm{A})^{-1} \mathrm{y} \text {. }
$$

O modelo básico de Leontief é classificado como de tecnologia baseada na indústria com enfoque indústriaindústria. A matriz dos coeficientes técnicos de produção foi gerada a partir das matrizes $\mathrm{V}$ e U, obtidas das agregações explicitadas anteriormente . Procedeu-se da seguinte forma.

Inicialmente definiu-se a matriz de coeficientes técnicos:

$$
\mathrm{B}=\mathrm{U}(\hat{X})^{-1 \mathrm{~b}}
$$

em que $\hat{X}$ é o vetor "X" diagonalizado.

Das TIPs podem ser obtidos ainda os vetores agregados E (22 x 1) (total da demanda final) e Q (22 x 1) (total da demanda: $Q=U+E$ ). Definiu-se então a matriz D (22 x 22), cujos elementos medem a fração da produção total do bem "j" produzido pelo setor "i" da seguinte forma:

$$
\mathrm{D}=\mathrm{V}(\hat{Q})^{-1}
$$

em que $\hat{Q}$ é o vetor “Q” diagonalizado. forma:

Os fluxos intersetoriais podem ser expressos na

$$
\mathrm{Q}=\mathrm{BX}+\mathrm{E}
$$

desde que $U=B X$. Sabendo-se que $X=V=D Q$, obtémse:

$$
\mathrm{Q}=\mathrm{BDQ}+\mathrm{E}
$$

executando operações matriciais relevantes, obtém-se:

$$
\mathrm{Q}=(\mathrm{I}-\mathrm{BD})^{-1} \mathrm{E}
$$

Nota-se que $\mathrm{Y}=\mathrm{DE}$. Esta relação transforma a demanda final por produto em demanda final setorial. Novamente, fazendo as operações matriciais relevantes, obtém-se a equação matricial final utilizada para calcular

\begin{tabular}{|c|c|c|c|}
\hline & Setor & & Atividade \\
\hline \multirow[t]{2}{*}{01} & Agricultura & 01 & Agropecuária \\
\hline & & 02 & Extrativa Mineral \\
\hline \multirow[t]{2}{*}{02} & Mineração & 03 & Extração de Petróleo e Gás \\
\hline & & 04 & Minerais Não-Metálicos \\
\hline & & 05 & Siderurgia \\
\hline \multirow[t]{2}{*}{03} & Metalsiderúrgico & 06 & Metalúrgia Não-Ferrosos \\
\hline & & 07 & Outros Metalúrgicos \\
\hline 04 & Maquinário & 08 & Máquinas e Tratores \\
\hline \multirow{2}{*}{05} & Eletroeletrônico & 10 & Material Elétrico \\
\hline & & 11 & Equipamentos Eletrônicos \\
\hline \multirow{3}{*}{06} & |Veículos & 12 & Automóveis, Caminhões e Ônibus \\
\hline & pretcuios s & 13 & Outros Veículos e Peças \\
\hline & & 14 & Madeira e Mobiliário \\
\hline \multirow[t]{2}{*}{07} & Florestal & 15 & Papel e Gráfica \\
\hline & & 16 & Indústria da Borracha \\
\hline & & 17 & Elementos Químicos \\
\hline \multirow[t]{2}{*}{08} & Petroquímico & 18 & Refino do Petróleo \\
\hline & & 19 & Químicos Diversos \\
\hline \multirow[t]{3}{*}{09} & Fármaco & 20 & Farmacêutica e Perfumaria \\
\hline & & 21 & Artigos de Plástico \\
\hline & Plástico s & 22 & Indústria Têxtil \\
\hline 10 & Flastico & 23 & Artigos do Vestuário \\
\hline & & 24 & Fabricação de Calçados \\
\hline & & 25 & Indústria do Café \\
\hline & & 26 & Beneficiamento de Produtos Vegetais \\
\hline & & 27 & Abate de Animais \\
\hline \multirow[t]{4}{*}{11} & Alimentícios & 28 & Indústria de Laticínios \\
\hline & & 29 & Indústria de Açúcar \\
\hline & & 30 & Fabricação de Óleos Vegetais \\
\hline & & 31 & Outros Produtos Alimentares \\
\hline \multirow[t]{6}{*}{12} & Diversos & 32 & Indústrias Diversas \\
\hline & & 33 & Serviços Industriais de Utilidade \\
\hline & & & Pública \\
\hline & & 34 & Construção Civil \\
\hline & & 35 & Comércio \\
\hline & & 36 & Transportes \\
\hline \multirow{7}{*}{13} & Servicos & 37 & Comunicações \\
\hline & & 38 & Instituições Financeiras \\
\hline & & 39 & Serviços Prestados às Famílias \\
\hline & & 40 & Serviços Prestados às Empresas \\
\hline & & 41 & Aluguel de Imóveis \\
\hline & & 42 & Administração Pública \\
\hline & & 43 & Serviços Privados Não-Mercantis \\
\hline
\end{tabular}
os multiplicadores:

$$
\mathrm{X}=(\mathrm{I}-\mathrm{DB})^{-1} \mathrm{Y}
$$

Quadro 1 - Agregação das atividades em setores Table 1 -Aggregation of the activities in sectors

Fonte: preparado pelo autor.

em que DB é a matriz dos coeficientes técnicos de produção. Chamando DB de A, tem-se:

$$
\mathrm{X}=(\mathrm{I}-\mathrm{A})^{-1} \mathrm{Y}
$$

Os valores da matriz A são determinados da seguinte forma: $\mathrm{T}_{12}=\mathrm{A}_{12} \mathrm{X}_{2} ; \mathrm{T}_{22}=\mathrm{A}_{22} \mathrm{X}_{2} ; \mathrm{T}_{23}=\mathrm{A}_{23} \mathrm{X}_{3} ; \mathrm{T}_{31}=\mathrm{A}_{31} \mathrm{X}_{1}$; e $\mathrm{T}_{11}=\mathrm{A}_{11} \mathrm{X}_{1}$. 
Desse modo, a matriz A é igual a:

$$
A=\left|\begin{array}{ccc}
A_{11} & A_{12} & 0 \\
0 & A_{22} & A_{23} \\
A_{31} & 0 & 0
\end{array}\right|
$$

O multiplicador de produto para um determinado setor é dado por:

$$
\left|\begin{array}{cccc}
A_{11} & A_{12} & \ldots & A_{1 n} \\
A_{21} & A_{22} & \ldots & A_{2 n} \\
\ldots & \ldots & \ldots & \ldots \\
A_{n 1} & \ldots & \ldots & A_{n n}
\end{array}\right| \cdot\left|\begin{array}{c}
1 \\
0 \\
\ldots \\
0
\end{array}\right|=A_{11}+A_{21}+\ldots+A_{n 1}=\sum_{i=1}^{n} A_{i 1} .
$$

O primeiro termo da expressão matricial descrita acima $(\mathrm{I}-\mathrm{A})^{-1}$ é a matriz dos multiplicadores de impacto de insumo-produto e o segundo é o vetor de demanda final, o que significa que para um incremento de uma unidade na demanda final de produtos do setor 1 esse setor pode crescer de $\Sigma \mathrm{A}_{\mathrm{i} 1}(\mathrm{i}=1, \ldots, \mathrm{n})$.

No caso das outras variáveis econômicas de interesse, como renda, emprego, impostos e importações, tem-se que uma mudança na necessidade de produto de um determinado setor causa mudanças na produção, na renda, no consumo de produtos importados, no recolhimento de impostos e no emprego. Conforme descrito, o impacto econômico na produção é estimado por:

$$
\Delta \mathrm{X}=(\mathrm{I}-\mathrm{A})^{-1} \cdot(\Delta \mathrm{Y}) \text {. }
$$

As mudanças nos níveis das outras variáveis econômicas são, então, calculadas pela multiplicação de seus coeficientes técnicos pela variação na produção $(\Delta X)$, resultante da injeção no consumo setorial. Considerando, por exemplo, o impacto na renda, tem-se como coeficiente de renda $r_{j}$ :

$$
r j=X_{s j} \div X_{j}
$$

em que $r_{j}=$ efeito direto da renda (coeficiente direto de renda); $\mathrm{X}_{\mathrm{sj}}=$ quantidade do valor do trabalho ao setor $\mathrm{j}$ para produzir $\mathrm{X}_{\mathrm{j}}$; e $\mathrm{X}_{\mathrm{j}}=$ valor bruto da produção do setor $\mathrm{j}(\mathrm{j}=1 \ldots . . \mathrm{n})$.

Dessa forma, o multiplicador de impacto na renda $\left(R_{I}\right)$ é $R_{I}=r_{j} \cdot \Delta X$.

O mesmo raciocínio pode ser estendido para as outras variáveis (impostos, importações, emprego etc).

\subsection{Decomposição da Matriz de Efeitos da MCS} tem-se:

Definindo $(\mathrm{I} \text { - A })^{-1}$ da equação básica (5) como $\mathrm{M}_{\mathrm{a}}$,

$$
\mathrm{X}=(\mathrm{I}-\mathrm{A})^{-1} \mathrm{Y}=\mathrm{M}_{\mathrm{a}} \mathrm{Y} .
$$

Essa expressão representa a renda setorial endógena como resultado das injeções dos multiplicadores contábeis. O cálculo das matrizes de efeitos (globais, transferência, cruzado e circular) é feito por meio da partição adequada da matriz $\mathrm{A}$, em duas outras matrizes, $\mathrm{B}$ e $\mathrm{C}$, de modo que $\mathrm{A}=\mathrm{B}+\mathrm{C}$.

$$
\begin{gathered}
\mathrm{A} \\
A=\left|\begin{array}{ccc}
A_{11} & A_{12} & 0 \\
0 & A_{22} & A_{23} \\
A_{31} & 0 & 0
\end{array}\right|=\left|\begin{array}{ccc}
A_{11} & 0 & 0 \\
0 & A_{22} & 0 \\
0 & 0 & 0
\end{array}\right|+\left|\begin{array}{ccc}
0 & A_{12} & 0 \\
0 & 0 & A_{23} \\
A_{31} & 0 & 0
\end{array}\right|
\end{gathered}
$$

O processo algébrico utilizado na decomposição do efeito multiplicador global, levando em conta as matrizes divididas B e C, é o descrito a seguir:

$$
\begin{aligned}
& X=A X+Y=(A+B-B) \cdot X+Y \\
& X=(A-B) \cdot+B \cdot X+Y \\
& X=(I-B)^{-1} \cdot(A-B) \cdot X+(I-B)^{-1} \cdot Y
\end{aligned}
$$

Fazendo D $=(\mathrm{I}-\mathrm{B})^{-1} \cdot(\mathrm{A}-\mathrm{B})$, tem-se que

$$
\mathrm{X}=\mathrm{D} \cdot \mathrm{X}+(\mathrm{I}-\mathrm{B})^{-1} \text {. }
$$

Este é o primeiro passo do processo.

Multiplicando a equação (8) por D e substituindo o valor encontrado (D.Y), tem-se que

$\mathrm{X}=\mathrm{D}^{2} \mathrm{X}+(\mathrm{I}+\mathrm{D}) \cdot(\mathrm{I}-\mathrm{B})^{-1} \cdot \mathrm{Y}$.

Este é o segundo passo do processo.

Analogamente, multiplicando a equação (8) por $\mathrm{D}^{2}$, substituindo o resultado $\left(\mathrm{D}^{2} \mathrm{X}\right)$ na equação (9) e isolando o valor de $X$, tem-se que

$$
\mathrm{X}=\left(\mathrm{I}-\mathrm{D}^{3}\right)^{-1} \cdot\left(\mathrm{I}+\mathrm{D}+\mathrm{D}^{2}\right) \cdot(\mathrm{I}-\mathrm{B})^{-1} \cdot \mathrm{Y}
$$

que é o terceiro e último passo do processo.

$$
\begin{aligned}
& \text { Fazendo } M_{a 1}=(I-B)^{-1} \\
& M_{a 2}=\left(I+D+D^{2}\right) \\
& M_{a 3}=\left(I-D^{3}\right)^{-1}
\end{aligned}
$$


Se a equação (10) for igualada à equação-padrão $X$ $=\mathrm{M}_{\mathrm{a}}$.Y, obtém-se a matriz de efeitos globais como o resultado de três outros efeitos: o efeito transferência $\left(\mathrm{M}_{\mathrm{a1}}\right)$, o efeito cruzado $\left(\mathrm{M}_{\mathrm{a} 2}\right)$, e o efeito circular $\left(\mathrm{M}_{\mathrm{a} 3}\right)$, ou seja:

$$
\mathrm{M}_{\mathrm{a}}=\mathrm{M}_{\mathrm{a} 3} \cdot \mathrm{M}_{\mathrm{a} 2} \cdot \mathrm{M}_{\mathrm{a} 1}
$$

A matriz de efeito transferência $\left(M_{a 1}\right)$ capta o efeito da transferência de insumos e produtos entre as atividades produtivas, semelhante ao multiplicador de impacto na produção da MIP.

A matriz de efeito cruzado $\left(\mathrm{M}_{\mathrm{2} 2}\right)$ capta as interações dentro e entre os três blocos de contas endógenas. Reflete os efeitos de mudanças exógenas na distribuição de renda sobre o produto e o emprego, sem considerar os efeitos de mudanças no emprego e no produto sobre a distribuição de renda.

A matriz de efeito circular $\left(\mathrm{M}_{\mathrm{a} 3}\right)$ assegura que o fluxo circular se completa entre as atividades endógenas, ou seja, os impactos de uma injeção original que fluem das atividades produtivas para o valor adicionado, deste para as instituições e de volta para as atividades produtivas.

\section{RESULTADOS E DISCUSSÃO}

Utilizando a MIP a preços básicos e aplicando os cálculos dos multiplicadores, obtêm-se os valores dos efeitos diretos que o aumento de uma unidade adicional na demanda final por produtos de cada setor causa na produção, nos salários, no emprego, nos impostos e nas importações, conforme o Quadro 2.

Comparando os valores dos multiplicadores de cada setor com a média geral, pode-se afirmar que os setores Florestal e Alimentícios obtiveram os melhores desempenhos, pois foram os únicos que apresentaram apenas um valor abaixo da média, especificamente no caso dos impostos e salários, respectivamente (Quadro 2).

A baixa contribuição em impostos se deve, principalmente, ao fato de os produtos florestais serem, em grande parte, básicos e semi-elaborados e de a carga tributária incidir mais sobre os produtos supérfluos, o que explica valores maiores para os setores Veículos e Eletroeletrônico sobre a participação e o potencial de arrecadação de impostos, em relação ao setor Florestal.

Uma das características mais importantes do setor Florestal diz respeito à remuneração salarial, pois além de oferecer postos de trabalho tanto no campo, como na indústria, a diferença salarial verificada entre o mais alto e o mais baixo é bem menor que a de outros setores, proporcionando melhor distribuição de renda.

Outro setor que apresentou multiplicadores significativos foi o Metalsiderúrgico, principalmente quanto aos efeitos sobre a produção. O multiplicador de produção do setor Florestal ( $5^{\circ}$ colocado) foi de 2,173, o que

Quadro 2 - Multiplicadores de impacto na produção, nos salários, no emprego, nos impostos e nas importações dos diversos setores em 1995

Table 2 - Impact Multipliers in production, wages, employment, taxes and imports of several sectors in 1995

\begin{tabular}{|l|c|c|c|c|c|}
\hline \multicolumn{1}{|c|}{ Setor } & Produção & Salário & Emprego & Imposto & Importação \\
\hline Agricultura & 1,666 & 0,181 & $\mathbf{0 , 2 3 4}$ & $\mathbf{0 , 0 3 9}$ & $\mathbf{0 , 0 4 0}$ \\
Mineração & $\mathbf{2 , 1 1 5}$ & $\mathbf{0 , 3 3 6}$ & 0,074 & 0,034 & $\mathbf{0 , 0 6 2}$ \\
Metalsiderúrgico & $\mathbf{2 , 4 6 3}$ & $\mathbf{0 , 3 3 2}$ & 0,065 & 0,032 & 0,122 \\
Maquinário & 1,841 & $\mathbf{0 , 3 3 4}$ & 0,047 & 0,022 & $\mathbf{0 , 0 8 6}$ \\
Eletroeletrônico & 2,048 & 0,278 & 0,047 & $\mathbf{0 , 0 3 7}$ & 0,190 \\
Veículos & $\mathbf{2 , 3 3 8}$ & $\mathbf{0 , 3 1 9}$ & 0,050 & 0,035 & 0,155 \\
Florestal & $\mathbf{2 , 1 7 3}$ & $\mathbf{0 , 3 4 2}$ & $\mathbf{0 , 0 9 8}$ & 0,029 & $\mathbf{0 , 1 0 1}$ \\
Petroquímico & 2,033 & 0,222 & 0,046 & $\mathbf{0 , 0 3 7}$ & 0,166 \\
Fármaco & 1,851 & 0,278 & 0,051 & 0,032 & 0,143 \\
Plásticos & $\mathbf{2 , 2 2 0}$ & $\mathbf{0 , 3 1 3}$ & $\mathbf{0 , 1 2 7}$ & 0,032 & 0,135 \\
Alimentícios & $\mathbf{2 , 4 2 6}$ & 0,270 & $\mathbf{0 , 1 4 7}$ & $\mathbf{0 , 0 4 5}$ & $\mathbf{0 , 1 0 3}$ \\
Diversos & 2,042 & $\mathbf{0 , 3 6 3}$ & 0,086 & 0,034 & $\mathbf{0 , 0 7 6}$ \\
Serviços & 1,545 & $\mathbf{0 , 4 3 9}$ & 0,087 & $\mathbf{0 , 0 5 2}$ & $\mathbf{0 , 0 3 8}$ \\
\hline \multicolumn{1}{|c|}{ Média } & 2,059 & 0,308 & 0,089 & 0,035 & 0,109 \\
\hline
\end{tabular}

Fonte: preparado pelo autor. 
significa que se a demanda final por produtos florestais aumenta $\mathrm{R} \$ 1.000$ ocorre uma variação em todos os setores da economia para atender a este aumento da demanda, de R $\$ 2.173$. No caso de emprego esta observação é diferente das demais, pois para cada $\mathrm{R} \$ 1.000$ de aumento na demanda gera-se aproximadamente 0,1 emprego, ou seja, um emprego a cada $\mathrm{R} \$ 10.000$.

Setores como Fármaco, Eletroeletrônico, Petroquímico, Maquinário e Veículos, tratados como a "meninados-olhos" por muitos governos, recebendo todo o tipo de incentivos como estratégia para favorecer o desenvolvimento regional, apresentaram uma quantidade maior de valores abaixo da média do que setores com melhor desempenho e menos incentivados, como os Alimentícios (agroindústria), Florestal e Agricultura.

No caso específico das importações, a análise que deve ser feita é a de que quanto menor o valor de seu multiplicador melhor, em parte, tende a ser a contribuição do setor para com a balança comercial, pois quanto mais a produção de um determinado setor aumenta menos matéria-prima importada ele precisa para elevar a produção, quando comparado com os demais. Este é o caso específico dos setores que utilizam fatores de produção e insumos intermediários do próprio país, como agricultura, agroindústria e florestal, e oposto ao caso dos setores Eletroeletrônico e Veículo, pois o aumento na sua produção implica aumento nas importações de mais matéria-prima, provocando um déficit na balança comercial, o que para um país carente em divisas como o Brasil seria um complicador para o seu balanço de pagamentos.

\subsection{Multiplicadores da MCS}

O Quadro 3 mostra que os multiplicadores da MCS são bem maiores que os multiplicadores da MIP, evidenciando uma grande contribuição dos modelos da MCS, em relação aos da MIP, por envolver também as contas sociais. Na MCS são consideradas as contas das instituições e do valor adicionado no cálculo dos multiplicadores, enquanto para os multiplicadores da MIP apenas a demanda intermediária é considerada.

Pode-se observar também que o setor Florestal, juntamente com os setores Mineração, Metalsiderúrgico, Alimentícios e Diversos, apresentou os valores dos multiplicadores acima da média. Por outro lado, setores como Eletroeletrônico, Petroquímico, Fármaco e Agricultura tiveram valores abaixo da média.

O multiplicador de efeito global do setor Florestal ( $6^{\circ}$ colocado) foi de 12,205, superando setores como Veículos, Eletroeletrônico, Maquinário, Fármaco, Plástico e Petroquímico, o que evidencia a importância estratégica desse setor para o Brasil.

Quadro 3 - Multiplicadores de impacto da MCS na produção das atividades, na renda das instituições e no pagamento do valor adicionado, em 1995

Table 3 - Multipliers of impact of SAM in the production of activities, institution income and payment of the added value, in 1995

\begin{tabular}{|c|c|c|c|c|}
\hline Multiplicador de Impacto & Global & Atividade & Instituição & Valor Adicionado \\
\hline Agricultura & 11,015 & 5,140 & 3,209 & 2,665 \\
\hline Mineração & 12,512 & 6,109 & 3,568 & 2,835 \\
\hline Metalsiderúrgico & 12,375 & 6,288 & 3,403 & 2,684 \\
\hline Maquinário & 11,908 & 5,700 & 3,449 & 2,759 \\
\hline Eletroeletrônico & 11,063 & 5,519 & 3,100 & 2,445 \\
\hline Veículos & 11,899 & 6,031 & 3,284 & 2,584 \\
\hline Florestal & 12,205 & 6,031 & 3,438 & 2,736 \\
\hline Petroquímico & 10,945 & 5,435 & 3,072 & 2,438 \\
\hline Fármaco & 11,204 & 5,431 & 3,213 & 2,560 \\
\hline Plásticos & 11,849 & 5,923 & 3,305 & 2,621 \\
\hline Alimentícios & 12,322 & 6,193 & 3,400 & 2,729 \\
\hline Diversos & 12,441 & 6,048 & 3,564 & 2,829 \\
\hline Serviços & 12,603 & 5,819 & 3,777 & 3,007 \\
\hline Média & 11,872 & 5,821 & 3,368 & 2,684 \\
\hline
\end{tabular}

Fonte: preparado pelo autor. 
Decompondo esses impactos globais em impactos sobre a produção das atividades, as rendas das instituições e a formação do valor adicionado, nota-se que o setor Florestal apresentou resultados favoráveis quanto a esses multiplicadores, principalmente para os das atividades e do valor adicionado. Tais resultados mostram que o aumento de $\mathrm{R} \$ 1.000$ na demanda das contas exógenas (conta capital e resto do mundo) desse setor implica um aumento total de $\mathrm{R} \$ 12.205$ sobre todo o fluxo circular da economia, sendo de $\mathrm{R} \$ 6.031$ o aumento na produção de todos os setores produtivos, de $\mathrm{R} \$ 3.438$ o aumento nas transferência às instituições (famílias, governo e empresas) e de $\mathrm{R} \$ 2.736$ o aumento nas remunerações do valor adicionado (capital, trabalho e impostos).

Além dos impactos sobre produção (atividades), instituições e valor adicionado, o multiplicador de efeito global pode ser decomposto em efeito transferência, cruzado e circular (Quadro 4).

O setor Florestal apresentou valor modesto para o efeito cruzado, quando comparado com os dos demais efeitos, mas, assim mesmo, ainda maior que o apresentado pelo setor Veículos e muito próximo ao do valor encontrado para o Eletroeletrônico.
O melhor desempenho do setor Florestal encontrase no efeito transferência, que é o mesmo valor encontrado pelo multiplicador da produção da MIP, visto que esse efeito capta a transferência entre os insumos e produtos dos setores produtivos, confirmando os ótimos índices de ligações para trás e para frente proporcionados pelo setor. O valor encontrado para o efeito circular mostra que esse setor tem um grande poder de fazer com que a renda dos fatores de produção proveniente do aumento da produção pelas atividades produtivas seja repassada às famílias e ao governo, e destes de volta ao processo produtivo.

\section{CONCLUSÕES}

De acordo com os resultados obtidos neste estudo, conclui-se que o setor florestal contribui significativamente para o desenvolvimento socioeconômico do País, tanto na participação da formação destes indicadores, quanto no impacto de seu crescimento, quando há aumento na demanda da economia.

Finalmente, cabe ainda ressaltar que se o IBGE agregasse de forma mais apropriada para o setor florestal, as

Quadro 4 - Decomposição dos multiplicadores de efeito global da MCS em efeitos transferência, efeitos cruzados e efeitos circulares, em 1995

Table 4 - Decomposition of the multipliers of global effect of SAM in transfer, effects crossed effects and circular effects, in 1995

\begin{tabular}{|l|c|c|c|}
\hline \multirow{2}{*}{ Setor } & \multicolumn{2}{|c|}{ Efeito } \\
\cline { 2 - 4 } & Transferência & Cruzado & Circular \\
\hline Agricultura & 1,666 & $\mathbf{2 , 2 9 1}$ & 2,037 \\
Mineração & $\mathbf{2 , 1 1 5}$ & 1,854 & 1,727 \\
Metalsiderúrgico & $\mathbf{2 , 4 6 3}$ & 1,608 & $\mathbf{2 , 5 7 8}$ \\
Maquinário & 1,841 & $\mathbf{2 , 2 1 3}$ & 1,878 \\
Eletroeletrônico & 2,048 & 1,769 & 1,776 \\
Veículos & $\mathbf{2 , 3 3 8}$ & 1,658 & 1,971 \\
Florestal & $\mathbf{2 , 1 7 3}$ & 1,758 & 1,843 \\
Petroquímico & 2,033 & 1,780 & $\mathbf{2 , 2 4 3}$ \\
Fármaco & 1,851 & $\mathbf{2 , 0 2 3}$ & 1,928 \\
Plásticos & $\mathbf{2 , 2 2 0}$ & 1,746 & 1,545 \\
Alimentícios & $\mathbf{2 , 4 2 6}$ & 1,484 & $\mathbf{2 , 1 9 0}$ \\
Diversos & 2,042 & $\mathbf{1 , 9 3 4}$ & $\mathbf{3 , 0 3 5}$ \\
Serviços & 1,545 & $\mathbf{2 , 4 9 3}$ & 2,107 \\
\hline \multicolumn{1}{|c|}{ Média } & 2,059 & 1,893 & \\
\hline
\end{tabular}

Fonte: preparado pelo autor. 
atividades e os produtos referentes à indústria florestal iria corroborar ainda mais os efeitos positivos do setor exposto neste estudo.

\section{REFERÊNCIAS BIBLIOGRÁFICAS}

CASTILHO, M. R. Algumas considerações sobre o uso de modelos computáveis de equilíbrio geral como

instrumento de análise do setor externo brasileiro. Rio de Janeiro: FUNCEX, 1994. 26 p. (Texto para discussão, 97).

FLICK, W. A.; TEETER, L. D. Multiplier effects of the southern forest industries. Forest Products Journal, v. 38, n. 11/12, p. 69-74, 1988.

MURRAY, B. C. Oligopsony vertical integration, and output substitution: welfare effects in U.S. pulpwood markets. Land Economics, v. 71, n. 2, p. 193-206, 1995.
NAJBERG, S.; RIGOLON, F. J. Z.; VIEIRA, S. P. Modelo de equilíbrio geral computável como instrumento de política econômica: uma análise de câmbio versus tarifas. Rio de Janeiro: BNDES, 1995. 33 p. (Texto para discussão, 30).

VALVERDE, S. R.; TEIXEIRA, E. C.; SILVA, M. L. Impactos da liberalização do comércio internacional nos produtos florestais da economia brasileira. Revista Árvore, Viçosa, v. 21, n. 2, p. 243-251, 1997.

VIEIRA, W. C. Notas sobre a construção de matrizes de contabilidade social. Revista Economia Rural, v. 9, n. 2, p. 30-37, 1998.

WEINTRAUB, A.; CHOLAKY, A. A hierarchical approach to forest planning. Forest Science, v. 37, n. 2, p. 439-460, 1991. 